\title{
'La felicità scivola tra le dita' di Abla Farhoud. Contributo alla traduzione della «scrittura migrante»
}

Elettra Bordino Zorzi

\section{(2) OpenEdition}

Journals

Edizione digitale

URL: http://journals.openedition.org/studifrancesi/34122

DOI: 10.4000/studifrancesi.34122

ISSN: 2421-5856

\section{Editore}

Rosenberg \& Sellier

\section{Edizione cartacea}

Data di pubblicazione: 1 novembre 2005

Paginazione: 338-351

ISSN: 0039-2944

\section{Notizia bibliografica digitale}

Elettra Bordino Zorzi, «'La felicità scivola tra le dita' di Abla Farhoud. Contributo alla traduzione della «Scrittura migrante»», Studi Francesi [Online], 146 (XLIX | II) | 2005, online dal 30 novembre 2015, consultato il 19 avril 2021. URL: http://journals.openedition.org/studifrancesi/34122 ; DOI: https:// doi.org/10.4000/studifrancesi.34122

\section{(c)}

Studi Francesi è distribuita con Licenza Creative Commons Attribuzione - Non commerciale - Non opere derivate 4.0 Internazionale. 


\section{'La felicità scivola tra le dita' di Abla Farhoud Contributo alla traduzione della «scrittura migrante»}

Tradurre si ricollega per via etimologica a trasportare, ossia portare da un luogo a un altro e per ciò stesso discostare, allontanare, insomma tradire, come proclama il celeberrimo adagio. Eternamente divisa fra un'ideale fedeltà e la chiarezza della riformulazione egocentrica, questa pratica millenaria conosce gli onori della ribalta innanzi tutto in epoca romantica, al tempo del risveglio delle coscienze nazionali, e ancora di più al giorno d'oggi, in un mondo intimamente sbriciolato, anche se ebbro di globalizzazione, dove i flussi migratori difficilmente arginabili generano nuovi rompicapi, puzzle etnici di ardua risoluzione. A testimonianza dell'interesse crescente per questo mestiere non di rado sminuito in passato, la discussione teorica, da sempre esistita, si è recentemente concretizzata in una disciplina autonoma, la traduttologia, fra precetti teorici e proposte operative, con una varietà di orientamenti che rispondono alle numerose sfide da affrontare.

Fra queste ci pare lecito annoverare una particolare forma di scrittura detta «migrante» o «immigrante» che, presentando un'originale conformazione linguistica, pone specifici problemi di traduzione. Si tratta, infatti, delle opere di un gruppo di scrittori contemporanei di origini diverse ma tutti residenti in Québec, che scelgono di esprimersi in francese, pur mantenendo con le lingue-culture di provenienza un legame profondo'. Di essi fa parte l'autrice teatrale Abla Farhoud, libanese di nascita, il cui primo romanzo, Le bonheur a la queue glissante, edito a Montréal dall'Hexagone nel 1998 e vincitore del premio France-Québec nel 1999, è stato da noi tradotto in italiano con il titolo La felicità scivola tra le dita, Roma, Sinnos, 2002 (premio di traduzione letteraria della città di Monselice «Leone Traverso - opera prima», 2003). Tale esperienza, con il suo inevitabile corteo di difficoltà e relative intuizioni, ci ha suggerito delle considerazioni che riteniamo possano contribuire utilmente al dibattito in corso.

\section{L'italiano, riflesso del francese}

Affrontare la traduzione di un testo significa, innanzi tutto, immergersi in un particolare universo di parole per trovare quell'unità «di lingua, di stile e di tono» ${ }^{2}$ indispensabile per la buona riuscita dell'impresa. I fattori che entrano in gioco sono molteplici e variamente combinati.

Nel nostro caso, utili indicazioni vengono fornite dalla riflessione metalinguistica che percorre l'opera. In essa è la protagonista, Dounia, a tessere il filo della narrazione raccontando in prima persona la sua lunga vita segnata dall'esperienza dell'emigrazione: il Libano, poi il Canada, poi ancora il Libano, infine il Canada, dove si stabilisce definitivamente con il marito e la numerosa prole. Il suo è un discorso risonante quasi esclusivamente nell'interiorità, un flusso di coscienza pacato e scor-

(1) Cfr. Y. GasquY-Resch, Littérature du Québec, Edicef-Aupelf, pp. 235-41; A. DE VAucher GraviLI (a cura di), D'autres rêves. Les écritures migrantes au Québec, Venezia, Supernova, 2000.
(2) Cfr. G. Mounin, Les belles infidèles, Paris, Les Cahiers du Sud, 1955, p. 149 e ss. Il principio dell'uniformità traduttiva viene, comunque, difeso da molti altri, soprattutto in ambito letterario. 
revole, con solo qualche lieve increspatura e accelerazione, una sequenza di pensieri che, seppur profondi, assumono una veste semplice, quasi elementare. Quest'anziana donna libanese, infatti, non dispone di un vocabolario ampio e variegato perché non sa né leggere né scrivere e parla solo l'arabo, senza nemmeno capire le due lingue ufficiali del paese d'adozione. Si ritrova così a dover chiedere sempre aiuto ai figli, chiamati a fungere da veri e propri interpreti. Ma questa situazione di dipendenza linguistica, lungi dall'apparirle normale, è per lei motivo di sofferenza, al punto da diventare uno dei nuclei tematici del racconto, disseminato di interrogativi su parole e silenzi, idiomi stranieri e comprensibilità.

Le bonbeur a la queue glissante si pone così immediatamente sotto il segno della pluralità linguistica. Redatto in francese, questo testo presuppone comunque l'esistenza di uno stadio anteriore, una specie di sostrato arabo. Tale bidimensionalità viene giustificata a livello diegetico con il ricorso alla tecnica della «mise en abîme»: la storia che Dounia dipana dentro di sé in arabo viene raccolta e trasformata artisticamente dalla secondogenita Myriam, scrittrice francofona $a^{3}$. Sul piano linguistico, la duplicità emerge chiaramente nel lessico finale, dove i proverbi libanesi di cui è costellato il romanzo vengono ripresi e elencati sia in francese che in arabo, e si manifesta in modo indiretto anche nelle poche battute che la protagonista rivolge ai nipoti:

«Maman toi, assis, toujours écrit, pour ça pas forte...» (p. 20) ${ }^{4}$.

Qui il francese mima la propria impenetrabilità, riportando le parole che un personaggio come Dounia avrebbe potuto «realisticamente» pronunciare. Per una resa ottimale, abbiamo forgiato un italiano altrettanto approssimativo e sgrammaticato, benché efficace dal punto di vista comunicativo:

«Mamma te seduta, sempre scrive, per questo non forte...» (p. 27).

Ma quali sono i tratti distintivi di questo francese, presumibilmente tradotto dall'arabo, in cui viene riportato il monologo interiore di Dounia? Dopo la scelta della lingua, una sola seppur «in contatto» con altre, un secondo elemento da considerare è il livello o registro adottato 5 . Il linguaggio di cui si serve Dounia è quello familiare parlato:

«Depuis quelques années, j’ai le temps de «philosopher», comme dit Salim pour me taquiner. Et pourquoi n'aurais-je pas le droit de «philosopher», j'ai vécu les trois quarts d'un siècle!

L'espace qu'il y a entre nous, je le vois surtout quand mes enfants sont avec leurs amis. Chaque fois, sans que j'y fasse attention, une question me revient: celle-là qui parle, celui-là qui rit dans une langue que je ne comprends pas, est-ce bien ma fille, est-ce bien mon fils? Est-ce que je suis bien sa mère?» (p. 83).

(3) Non ci interessa in questa sede l'eventuale fondatezza di richiami autobiografici che pur sono frequenti nella letteratura «migrante».

(4) Le pagine delle citazioni sono quelle delle due edizioni, francese e italiana, menzionate sopra.

(5) Per la spiegazione di «lingue in contatto», «livello o registro» e altri termini utilizzati in seguito, che denotano concetti ormai imprescindibili della disciplina sociolinguistica, rinviamo, oltre che ai
«Da qualche anno ho tempo di «filosofare», come dice Salim per punzecchiarmi. E perché poi non avrei il diritto di «filosofare», io che ho vissuto tre quarti di secolo!

La distanza che c'è tra me e i miei figli, la noto soprattutto quando si trovano con i loro amici. Ogni volta, senza che me ne renda conto, mi rifaccio sempre la stessa domanda: Quella che parla, che scherza in una lingua che non capisco, è davvero mia figlia, è davvero mio figlio? Sono davvero io loro madre?» (p. 83).

classici del settore (p.e. URIEL WeINRICH, Languages in contact, New York, 1953, William Labov, Sociolinguistique, Paris, Minuit, 1976 e JosHUA A. Fishman, Sociolinguistics: a brief introduction, Rowley (Massachussetts), Newbury House, 1970), a saggi di carattere divulgativo: JULIETTE GARMADI, La sociolinguistique, Paris, PUF, 1981; L.-J. CAlvet, La sociolinguistique, Paris, PUF, («Que sais-je?»), 1993. 
Frasi brevi, soprattutto coordinate, con qualche esclamativa e più numerose interrogative, frasi, però, che non indulgono mai in una sintassi sciatta o marcatamente popolare, ma anzi risultano sempre improntate a una certa dignità formale, sia nei passi narrativi e di commento che negli scambi dialogici:

«J'aimerais écrire un livre sur toi, mère» (p. 29).

«Dans notre culture, on ne dit pas "tu mens" à sa mère, mais je sentais que c'est ce qu'elle voulait dire» (p. 30). $\ll$ Mi piacerebbe scrivere un libro su di te, madre mia» (p. 36).

«Nella nostra cultura, non si dice alla propria madre "stai mentendo", ma sentivo che era questo che voleva dire» (p. 37).

I nipoti e i figli si rivolgono sempre alla protagonista con amore e rispetto e quest'ultimi la chiamano addirittura, con deferenza, «madre mia».

Analogamente il lessico è quello corrente d'uso quotidiano, ma privo di eccessi idiomatici e di elementi di argot, nonché impermeabile alle mode del momento, una caratteristica che è coerente con il personaggio di Dounia. Questa, difatti, avendo lasciato il contesto linguistico di appartenenza, resta indifferente alle tendenze di punta del linguaggio, in patria come all'estero. Tipicamente popolare, invece, è la modalità stilistica che privilegia il riferimento alla fisicità del mondo e del corpo anche nelle descrizioni di sentimenti o stati d'animo:

«Une main en acier me serrait la gorge et une pierre lourde me pressait la poitrine. Pendant toute la journée. Sans répit» (p. 31).
«Una mano di acciaio mi stringeva al collo e un macigno mi gravava sul petto. Tutto il giorno. Senza tregua» (p. 38).

Infine, dopo il registro, vanno osservate le particolarità idiolettali:

«Je ne suis pas très bonne en mots. Je ne sais pas parler. Je laisse la parole à Salim. Moi, je donne à manger.

Mes mots sont les branches de persil que je lave, que je trie, que je découpe, les poivrons et les courgettes que je vide pour mieux les farcir, les pommes de terre que j'épluche, les feuilles de vigne et les feuilles de chou que je roule» (p. 14).
«Non sono molto brava con le parole. Non so parlare. Lascio la parola a Salim. Io do da mangiare.

Le mie parole sono i rametti di prezzemolo che lavo, scelgo, taglio, i peperoni e le zucchine che svuoto per poterli farcire meglio, le patate che sbuccio, le foglie di vite e di cavolo che arrotolo» (p. 22).

Le parole di Dounia sono prive di ornamenti, custodite nell'intimità e circondate dal silenzio. A volte si fanno gesto concreto, addirittura cosa, in contrapposizione a un marito ciarliero e a una fase giovanile di maggiore apertura e spigliatezza. Un ultimo aspetto è degno di nota:

«Ceux qui vont très longtemps à l'école [...] disent en vingt mots ce que l'on pourrait dire en trois ... Le meilleur des mots, un seul... C'est ce que je pense» (p. 25).
«Chi va tanto a scuola $[\ldots]$ Dice venti parole quando ne basterebbero tre... Una parola sola, la migliore... Ecco come la penso» (p. 32). 
Il modo di esprimersi della narratrice intradiegetica è dunque intenzionalmente essenziale, senza vezzi né concessioni estetiche, frutto di uno stile personale che trae il suo vigore dall'esperienza di tutti i giorni, filtrata attraverso il buon senso e una saggezza di matrice popolare.

Possiamo allora concludere che il francese basilare ma limpido del libro Le bonbeur a la queue glissante è una lingua piana, spogliata di singolarità troppo vistose, per svolgere una funzione eminentemente veicolare; è una componente fondamentale di quell'idioma standard a vocazione universale che costituisce il patrimonio comune di nazioni - quelle francofone - anche molto distanti fra loro; è, su un piano più propriamente intralinguistico, il medium diretto del dialogo con se stessi. Parallelamente, nella nostra versione abbiamo cercato di mettere a punto un italiano neutro e trasparente, ovverosia quello, scevro da inflessioni regionali e coloriture gergali, che risulta comprensibile a tutti i parlanti, evitando fughe verso l'alto, verso forme troppo auliche o ricercate, ma anche sbavature eccessivamente colloquiali'. Particolare attenzione è stata rivolta in primo luogo alla struttura sintattica, soprattutto quando si piega a un andamento emotivo:

«Lui qui aime tant discuter avec les étrangers, pourquoi ne discute-t-il jamais avec moi? Avec les autres, il écoute et donne son opinion, avec moi, il n'entend pas ce que je dis et, de toute façon, c'est toujours lui qui a raison! Autant parler à un mur...» (p. 61).
«Con gli estranei gli piace tanto discutere, perché allora con me non lo fa mai? Con gli altri ascolta e dice la sua, con me invece non sta a sentire quel che dico e comunque ha sempre ragione lui! Tanto vale parlare al muro...» (p. 63).

Per raggiungere lo stesso risultato espressivo del francese, che qui comunica stizza e irritazione, è necessario in italiano individuare il giusto ordine delle parole, appoggiandosi anche a puntelli sintattici, quali gli avverbi «allora» e «invece», che di fatto mancano nell'originale. Per ragioni di equilibrio interno, poi, è bene non abusare delle forme enfatiche di derivazione francese del tipo «è lui che...», preferendo piuttosto il costrutto con il soggetto dopo il verbo. Il testo finale, insomma, secondo un tradizionale procedimento traduttivo, deve sembrar concepito e redatto nella lingua d'arrivo.

A volte, l'organizzazione delle frasi si complica, arricchendosi di echi sonori che fanno sospettare l'ingerenza, squisitamente letteraria, dell'autrice:

«Je creuse et je creuse ce qui me reste dans le creux de ma mémoire, espérant trouver un jour la paix dans cette tête pleine de trous et de crevasses» (p. 119).
«Scavo e scavo quel che resta nell'incavo della memoria, sperando di trovare un giorno la pace in questa testa piena di vuoti e incrinature» (p. 115).

Il ruolo cruciale svolto qui dai significanti dovrebbe trasparire nella versione italiana grazie alla ripetizione delle stesse radici lessicali («Scavo e scavo», «nell'incavo») e degli stessi fonemi (cfr. «resta», «questa testa»), soprattutto [s], [t] e [r], liquida che si ritrova due volte anche nel vocabolo «incrinature», a risonanza onomatopeica.

Altrove l'intervento della Farhoud è ancora più massiccio, l'assetto sintattico ancora più sofisticato, in quanto si fonda sulla replicazione di uno o più moduli costruttivi. Fra i vari passi in cui questo avviene, segnaliamo quello a più alta densità formale:

(6) La gerarchia qui prospettata è relativa alle opzioni a nostro parere necessarie per realizzare al meglio questa traduzione, non certo a un giudizio di valore sulle molteplici varianti linguistiche. 
«Il y a des choses que l'on ne peut pas dire, que l'on ne dit pas, même pas à soi-même, des choses que l'on voudrait enfouir loin.

Il y a des choses qui remontent malgré soi, comme une vomissure, et que l'on ravale avec aigreur et amertume, il y a des violences que l'on ne peut pardonner, des violences que l'on voudrait prendre pour de la démence, des absences de raison que l'on ne peut pardonner, ni oublier.

Il y a des choses qui ont le poids de toutes nos peurs rassemblées, de toutes nos lâchetés.

Il y a des choses que l'on ne peut ni raconter ni dire à voix basse tant on en a honte. Il y a des hontes qui ne peuvent s'apaiser avec le temps, que l'on ne peut se pardonner, ni oublier. Des hontes qui restent intactes comme si on venait de les vivre.

J'ai honte... Depuis cinquante années, j'ai honte. Même y penser, j'ai honte» (p. 147).
«Ci sono cose che non si possono dire, che non si dicono, nemmeno a se stessi, cose che vorremmo nascondere sottoterra.

$\mathrm{Ci}$ sono cose che ritornano a galla nostro malgrado, come un rigurgito, e che rimandiamo giù con astio e amarezza, ci sono violenze che non si possono perdonare, violenze che vorremmo prendere per follia, pazzie che non si possono perdonare né dimenticare.

Ci sono cose che pesano come tutte le nostre paure messe insieme, come tutte le nostre vigliaccherie.

$\mathrm{Ci}$ sono cose che non si possono raccontare né dire a bassa voce, tanta è la vergogna. $\mathrm{Ci}$ sono onte che non smettono di bruciare con il tempo, che non si possono perdonare né dimenticare. Onte che restano intatte come se le avessimo appena subite.

Mi vergogno... Da cinquant'anni, mi vergogno. Al solo pensiero, mi vergogno» (p. 140).

Questo incipit di uno degli ultimi capitoli, in cui la voce del personaggio principale riprende il sopravvento solo nell'ultimo paragrafo, si configura come un'unità testuale a sé stante non solo visivamente, in quanto delimitato da una riga bianca che lo separa da quel che segue, ma anche retoricamente, in quanto in esso ricorrono le stesse parole, gli stessi costrutti, ora semplicemente reduplicati, ora invece imbricati in modo diverso, secondo il procedimento della variatio. In questo microsistema conchiuso in sé stesso, concordanze e opposizioni si intrecciano generando un ritmo accentuato, martellante, quasi un canto di lamentazione. Come ricreare tutto questo in italiano? Innanzi tutto rispettando la scansione tipografica e la punteggiatura dell'originale, come ci impegniamo peraltro a fare sempre, se possibile. Poi risolvendo l'impiccio del pronome «on», inesistente in italiano: lo traduciamo con il «si» passivante o con il «noi» a seconda della cadenza che acquista la frase nel nostro testo e che ci sforziamo di rendere simile a quella francese. In funzione di questo obiettivo, curiamo naturalmente le ripetizioni, soprattutto quelle in posizione di giunto sintattico (il «che» relativo un po' ovunque, il «come» del secondo paragrafo) e puntiamo alla fluidità dell'insieme cambiando registro, dove necessario, anche se di poco: qui più familiare («remontent»-«venire a galla», «avaler»-«mandar giù»), lì più letterario («tanta è la vergogna» piuttosto che «tanto ci vergogniamo»,«Même y penser»-«Al solo pensiero»), purché ogni sfasatura venga opportunamente compensata ${ }^{7}$.

L'esigenza di ricostruire un italiano fluente e immediato si impone ovviamente anche su scala inferiore. Dal punto di vista morfologico, seguiamo un uso non corretto che trova tuttavia legittimazione in una prassi orale ormai consolidata, cui si sono conformati alcuni dizionari":

«C'est surtout pour Abdallah que je m'inquiète. Qui s'occupera de lui quand son père et moi ne serons plus là? Si mes enfants sont prêts à envoyer leur mère à l'hospice, laisseront-ils leur frère dans la rue?» (p. 18).

(7) La «compensazione» è una delle risorse del traduttore. Cfr., ad esempio, Peter Newmark, La traduzione: problemi e metodi, Milano, Garzanti,
«A preoccuparmi è soprattutto Abdallah. Chi si prenderà cura di lui quando io e suo padre non ci saremo più? Se i miei figli sono pronti a spedire in ospizio loro madre, lasceranno loro fratello in mezzo a una strada?» (p. 25).

1988 , p. 66.

(8) Cfr. lo Zingarelli del 1994. 
In virtù di un'estensione della norma grammaticale, l'aggettivo «loro», come gli altri possessivi di fronte a nomi di parentela al singolare, comporta l'omissione dell'articolo determinativo. Invece ci pareva più ardita grammaticalmente, e quindi un po' più bassa rispetto al livello fissato, la sostituzione del pronome complemento di termine «loro» con il singolare «gli», sebbene quest'ultimo sia ormai prevalente all'orale e comunque legittimato dall'uso che ne hanno fatto alcuni grandi scrittori fra cui Cesare Pavese. Ci era tuttavia possibile non incorrere in sgradevoli pesantezze, grazie alla bassa frequenza del problema e all'impiego di specifici accorgimenti. Ecco uno dei rari esempi incontrati:

«Je regrettais le temps où j'avais seulement à leur donner à manger pour les nourrir, mes fils avaient besoin d'autre chose, mais je ne savais pas quoi. [...] Les valeurs que nous essayions de leur transmettre, parfois par la force, devenaient ridicules à leurs yeux. L'extérieur était plus attirant que tout ce que nous pouvions leur offrir» (pp. 112-13).
«Rimpiangevo il tempo in cui dovevo solamente dar loro da mangiare per farli crescere: i mei figli avevano bisogno d'altro, ma non sapevo di cosa. [...] I valori che cercavamo di trasmettere loro, a volte con la forza, erano per loro ridicoli. Il mondo di fuori li attirava più di tutto quello che potevamo offrire noi» (pp. 108-09).

Il troncamento dell'infinito, un'adeguata disposizione delle parole e, ove possibile, la vera e propria omissione fanno passare inosservata questa forma avviata all'estinzione, ma di buona tenuta letteraria. In un solo punto, ci pare, la strategia adottata ha comportato una perdita espressiva:

«Je ne pouvais pas grand-chose pour eux, mais je pouvais au moins leur donner à manger et laver leurs vêtements» (p. 32).
«Non potevo far molto per loro, ma potevo almeno prepar da mangiare e lavare i panni» (p. 38).

Il testo italiano sacrifica alla scioltezza dell'insieme la focalizzazione del francese sui figli, chiamati in causa da «eux», «leur» e «leurs».

La ricerca di un linguaggio limpido e lineare continua sul piano lessicale, nella scelta del termine più appropriato, ma soprattutto nella traduzione delle locuzioni, per le quali viene applicata la tecnica, ben nota, dell'equivalenza:

«Même si tous les démons de l'enfer s'étaient donné le mot pour nous rendre la vie invivable, jamais je n'aurais dû accepter de partir de chez elle dans ces conditions. Quand je repense à tout cela aujourd'hui, je me dis que ma belle-mère ne devait pas être saine d'esprit pour faire une chose pareille: mettre toute une famille à la porte! Quand le coeur d'une personne commence à durcir on ne sait pas jusqu'où cela peut aller» (p. 67).
«Anche se tutti i diavoli dell'inferno si fossero passati parola per renderci la vita impossibile, mai e poi mai avrei dovuto accettare di lasciar casa sua in quelle condizioni. Quando mi capita di ripensarci, dico tra me e me che mia suocera non doveva essere sana di mente per fare una cosa simile: sbattere fuori un'intera famiglia! Quando una persona comincia a diventare dura di cuore, non si sa fin dove può arrivare» (p. 68).

Ne La felicità scivola tra le dita, tutti i modi di dire appartenenti al francese standard vengono resi con le corrispondenti espressioni italiane, come risulta chiaramente dal confronto dei termini qui evidenziati in grassetto.

Il metodo traduttivo fin qui sostenuto, che prevede la riformulazione dell'originale secondo i dettami della lingua d'arrivo, è invece del tutto inadeguato in quei 
pochi casi in cui il linguaggio sobrio della protagonista cede alla tentazione della figuratività, attuando propri scarti stilistici. Capita, infatti, di imbattersi in similitudini e metafore di nuovo conio, attribuibili a Dounia, alla sua forma mentis:

«Je regarde ma fille écrire. Ses doigts sont comme des mouches enfermées dans un bocal, ils voltigent et reviennent toujours à la même place» (p. 27).
«Guardo mia figlia scrivere. Le sue dita assomigliano a delle mosche rinchiuse in un barattolo, volteggiano ritornando sempre allo stesso posto» (p. 34).

Le dita della figlia, che volteggiano sulla tastiera del computer, sono paragonate a delle mosche rinchiuse in un barattolo. Per non alterare l'incisività del testo francese, è allora bene discostarsene il meno possibile, cercando di restituire, oltre al contenuto, la forma, la sostanza concreta di cui si compone l'immagine. L'umano e il volatile confluiscono ancora più avanti, in un'amara constatazione:

«Mes gestes tombent dans le vide comme des oiseaux morts» (p. 63).
«I miei gesti cadono nel vuoto come uccelli morti» (p. 64).

Anche qui, massima fedeltà all'originale, le cui potenzialità espressive devono rimanere intatte. Un'ultima citazione:

«[...] mon âme et mon cœur ont six morceaux, non, pas morceaux mais branches. J'ai un cœur à six branches et avec mes cinq petits-enfants ça donne onze branches» (p. 106). $\ll[\ldots]$ il mio cuore e la mia anima si dividono in sei parti, anzi non parti ma rami. Ho un cuore a sei rami e con i miei cinque nipoti fanno undici rami» (p. 103).

La collisione semantica fra cuore e albero riattiva un simbolismo vitalistico di lunga data, che va rievocato nel testo d'arrivo calcandolo su quello di partenza.

Se escludiamo il tipo di casi appena illustrato, è allora possibile trarre una prima conclusione: l'italiano de La felicità scivola tra le dita aspira innanzi tutto a divenire il «vetro trasparente»" del francese, ossia a rifletterlo ma per mezzo di una superficie invisibile, perché l'«altro da sé» va completamente assorbito, assoggettato alle proprie leggi, sintattiche, fonetiche e lessicali. Insomma, l'idioma straniero sfuma e svanisce, lasciando dietro di sé solo il proprio «spirito» disincarnato, senza l'impronta tangibile della sua lettera.

\section{Le altre lingue}

Ma, come già anticipato, Le bonbeur a la queue glissante non si esaurisce nel riferimento a un solo sistema linguistico e culturale, quello francese; al contrario, si inscrive esplicitamente in una realtà bilingue, il Québec, dove si parla anche l'inglese. Questa lingua, però, che pur occupa in quello stato canadese una posizione di prestigio, in quanto dominante nel mondo degli affari, all'interno dell'opera da noi tradotta rimane sullo sfondo, relegata in qualche nome proprio o titolo, che lasciamo come 
nell'originale (cfr. la trasmissione «Father knows best» e l'attore «Jacky Gleason», rispettivamente pp. 91-92 e p. 90), e in un sostantivo di diffusione internazionale («cow-boy», p. 69, di cui viene leggermente cambiata la grafia in «cowboy», p. 70). Esiste, però, un problema di interferenza che si manifesta nella concordanza dei modi e dei tempi verbali:

«Ce soir-là, sa maison lui parut grande, même immense, et ses soucis bien petits.

Le malheur, c'est qu'Abdo le malheureux s'est très vite habitué à son bonheur... Parfois, quand j'entends quelqu'un se plaindre, moi y compris, je pense à cette histoire $[\ldots] \gg($ p. 49).
«Quella sera, la sua casa gli parve grande, addirittura immensa, e ben misere le sue preoccupazioni.

Il guaio è che Abdo l'infelice si abituò molto presto alla felicità... A volte, quando sento lamentarsi qualcuno, me compresa, penso a questa storia $[\ldots] \gg($ p. 53).

Il «passé composé» sembra qui ricalcare il «present perfect» piuttosto che conformarsi all'usuale organizzazione verbale francese. A ogni modo, la nostra versione segue sempre le leggi della consecutio italiana.

Un altro tipo di caratterizzazione linguistica deve essere, invece, secondo noi conservata e valorizzata perché, anche se limitatamente presente, concorre in modo palese alla ricchezza dell'opera:

«Des gendres, brus, chums ou blondes, comme ils disent, le mari de Samira est le seul avec qui nous pouvons parler notre langue...» (p. 132).
«Tra generi, nuore, chums e "bionde", come dicono loro, il marito di Samira è l'unico con cui possiamo parlare la nostra lingua...» (p. 127).

La varietà quebecchese, tassello importante del mosaico francofono, viene qui evocata attraverso un prestito e un calco che abbiamo rigorosamente mantenuto, dandone poi in nota la traduzione boyfriend e «morose» che, in quanto costituita da un anglicismo e da un termine regionale, risulta avere nel testo d'arrivo un effetto analogo.

In base allo stesso principio sono stati fedelmente trascritti i nomi di persona e di luogo e a maggior ragione «Montréal», il cui accento assume rilevanza politica all'interno della questione identitaria, in quanto segno di appartenenza alla comunità francofona e, per ciò stesso, strumento di rivendicazione della coscienza nazionale quebecchese.

In un altro brano, è il tessuto metaforico a subire l'influenza dell'ambiente circostante:

$\ll[\ldots]$ Ils ont toujours aussi frileux, frileux comme des pingouins».

«C'est qu'il protègent leurs petits. Qu'est-ce qu'ils font d'autre, les immigrants? Ils se collent les uns sur les autres pour ne pas laisser entrer le froid».

$$
[\ldots]
$$

$\ll[\ldots]$ Si nous, nous avons perdu notre pays, eux, ils n'en ont pas encore... y a de quoi avoir froid...» (p. 52).
«[...] Questi quebecchesi tremano sempre come allora, tremano come pulcini».

«E che vogliono proteggere i loro piccoli. Cos'altro fanno gli immigrati? Si stringono gli uni agli altri per non lasciar entrare il freddo».

[...]

«[...]Se noi abbiamo perso il nostro paese, loro non ne hanno ancora uno, c'è di che tremare...» (p. 56). 
Qui, però, abbiamo preferito rinunciare all'icasticità dell'originale per ricreare, con una locuzione del tutto italiana, l'ambivalenza semantica di figure che vertono sul freddo per alludere anche alla paura. Una nota a pie' di pagina, tuttavia, avverte dello scollamento fornendo come raffronto una traduzione letterale.

Ma se il quebecchese non fa che circoscrivere e qualificare, seppur a tratti, quel francese standard eletto, come abbiamo visto, a mezzo espressivo, esiste nel romanzo un altro polo linguistico e culturale che non è semplicemente fonte di attrazione, ma autentico vettore di straniamento: la civiltà mediorientale incarnata dal Libano.

Anche in questo caso, la traduzione deve rimanere il più possibile aderente al testo di partenza. I nomi dei personaggi sono stati pedissequamente ricopiati, senza nemmeno adattare il dittongo francese «ou» alla dizione italiana, per rispettare la volontà dell'autrice che desiderava alimentare la dissonanza araba con quella di una lingua ugualmente «altra», anche se a noi più vicina. Così si legge ad esempio «Dounia» (sarebbe piuttosto «Dunia» in italiano), che significa il cosmo, come viene precisato a p. 99 (p. 97 dell'edizione italiana). Lo stesso vale per i prestiti, segnati generalmente in corsivo, che peraltro sono talmente pochi da poter essere enumerati: il vocativo sitto, nonna, il piatto tradizionale hoummos bi tahini, l'appellativo Oum Abdallah, madre di Abdallah, spiegato in nota.

Ma la vera origine di quell' «estraneità» che permea tutta l'opera, costituendo uno dei suoi aspetti di maggior fascino, è la particolare visione del mondo suggerita dai proverbi libanesi disseminati nel romanzo. Questi vengono citati senza virgolette né altri espedienti demarcativi, per cui si inseriscono nella narrazione in sordina, quali punti di condensazione del pensiero che solo in essi trova delle certezze. Ricompaiono, poi, nel lessico finale che li raggruppa tutti proponendone anche di nuovi ${ }^{10}$, mai incontrati prima. L'elenco così formato è impreziosito dall'inclusione dei detti originali in arabo. Riguardo a questi, per nostra curiosità e per scrupolo ci siamo avvalsi della consulenza di due esperti della lingua, ma la nostra versione si basa esclusivamente sul testo francese, frutto della rielaborazione artistica di Abla Farhoud e, quindi, vera sostanza del libro ${ }^{11}$.

I proverbi sono complessivamente cinquantuno, raccolti in appendice quasi per sottolineare che la loro è una diversità manifesta, dichiarata, di cui il traduttore non può non tener conto:

«Un paysan qui se suffit à lui-même est un sultan qui s'ignore...» (p. 9).
«Un contadino che basta a se stesso è un sultano e non lo sa...» (p. 17).

Questo primo detto dà un'idea del metodo che ci è parso sin da subito il più idoneo: riprendere gli stessi lessemi, per assaporare la «lettera», ma disporli in una forma armoniosa, che risulti perfettamente italiana, per scongiurare ogni rischio di pedissequo «mot à mot». Per questo, la seconda relativa viene trasformata in una

(10) Dei primi verrà indicata solo la collocazione all'interno del romanzo, dei secondi, per forza di cose, quella nella sezione finale. Per uniformità di grafia, sopprimiamo gli eventuali puntini prima e dopo la massima popolare.

(11) Ciò non toglie che l'apporto dei colleghi sia stato utilissimo, se non indispensabile, almeno in un'occasione:

«Le chagrin n'est séparé de la joie que par le temps de tourner une feuille» (p. 175).
«Il dolore dista dalla gioia solo il tempo di voltare una foglia» (p. 162).

Il sostantivo francese «feuille» ha due significati: «foglio»e «foglia». La conoscenza del testo arabo ci ha consentito di sciogliere l'ambiguità a vantaggio di un'interpretazione per la quale eravamo già propensi, vista la natura essenzialmente orale della cultura qui evocata. 
coordinata che, non di rado, serve nella nostra lingua per designare l'inconsapevolezza di qualcosa di positivo ${ }^{12}$.

$\mathrm{Ma}$ le scelte or ora precisate si giustificano anche con considerazioni relative al ritmo delle frasi, componente fondamentale che in alcuni casi si rivela essere la costrizione maggiore:

«Même l'épée n'arrivera pas à le toucher si les mots n'y sont pas arrivés» (p. 61).

«N'enseigne pas à ton enfant, le destin s'en charge» (p. 106).

$\gg$ Les coups du destin sont plus nombreux que tout ce qui pousse sur terre» (p. 175).
«Nemmeno la spada potrà colpirlo, se non ci sono riuscite le parole» (p. 63).

«Non dare insegnamenti a tuo figlio, lo farà il destino» (p. 104).

«Più numerosi di tutto quel che cresce sulla terra sono i rovesci del destino» (p. 162).

Per tentare di riprodurre la cadenza delle varie massime, prevalentemente basata su assonanze e ripetizioni, abbiamo spesso fatto appello alla costruzione simmetrica, tipicamente italiana, con il soggetto al termine della proposizione. Ma la partitura del testo francese può essere ricreata anche per analogia, imitando le strutture attestate nel nostro patrimonio di sentenze. Per esempio: (p. 23)

«Une main vide est une main sale $[. .]$. (p. 30)

«Mano vuota, mano sporca [...]»

La traduzione di questo proverbio si modella sull'adagio «gioco di mano, gioco di villano». Naturalmente bisogna salvare il più possibile anche gli effetti sonori:

«Si jeunesse revenait un jour, je lui raconterais ce que vieillesse a fait de moi» (p. 11).
«Se un giorno ritornasse giovinezza, le racconterei cos'ha fatto di me vecchiezza» (p. 19).

Il ricorso al termine «vecchiezza», sinonimo attenuato e più nobile di «vecchiaia», ci permette di non lasciar cadere la rima interna, conferendo al testo quel decoro formale che in francese gli deriva soprattutto dalla soppressione dell'articolo davanti ai due sostantivi principali. Altrove il mantenimento delle corrispondenze fonetiche è facilitato dalla stretta parentela fra le due lingue, per cui ci limitiamo a qualche ritocco sintattico: 174).

«La corde du mensonge est courte» (p.

«Dieu donne des noix à ceux qui n'ont plus de dents» (ibid.). (p. 161).

«Corta è la corda della menzogna» (p. 162).

La traduzione di questi due detti prova la fecondità dell'approccio traduttivo scelto: gli equivalenti italiani «Le bugie hanno le gambe corte» e «Chi ha i denti non ha il pane e chi ha il pane non ha i denti» risulterebbero alla lettura senz'altro meno

(12) Si pensi ad affermazioni comuni del tipo: è bello e non lo sa, è molto fortunato e non lo sa. 
significativi della versione proposta, che, proprio perché letterale, si fa portatrice della diversità, anche se la esprime in una veste perfettamente legittima nella lingua d'arrivo.

Piccoli assestamenti sono comunque sempre necessari, come succede con il possessivo, molto più frequente in francese:

«Chacun sème son verger avec sa propre intelligence» (p. 69).
«Ognuno semina il proprio orto con l'intelligenza che si ritrova» (p. 70).

Il «verger» diventa qui «orto» perché il frutteto orientale è, in senso figurato, il giardino interiore. Altrove lo stesso genere di problema ha richiesto una rielaborazione sintattica più radicale:

«Seuls tes ongles gratteront ta peau en te soulageant» (p. 35).
«Solo grattandoti con le tue unghie troverai sollievo» (p. 41).

Come scontato in questo tipo di attività, il passaggio da una lingua all'altra comporta dei guadagni e delle perdite. Cominciamo dai primi:

«On dit qu'il est impossible de se dissimuler quand on est amoureux, enceinte ou monté sur un chameau» (p. 131).
«Dicono che sia impossibile non farsi notare quando si è innamorati, in gravidanza o in groppa a un cammello» (p. 125).

Qui, una difficoltà morfologica si risolve in un incremento della sonorità. Come già accennato in precedenza, l'indefinito «on» trova diversi corrispettivi in italiano. In questo periodo, il primo viene tradotto con una terza persona plurale e il secondo con il «si» impersonale che, però, può essere solo maschile plurale, mentre il pronome francese accetta l'accordo sia al singolare che al femminile. Tale discrepanza obbliga a delle modifiche che vanno ad aggiungersi a quella, già effettuata nella proposizione principale, della modulazione ${ }^{13} \mathrm{di}$ «se dissimuler» in «non farsi notare». Il risultato finale, comunque, ci pare soddisfacente: opportunamente costruita, la subordinata temporale diventa più ricca sul piano del significante, fregiandosi di numerose allitterazioni (innamorati, in gravidanza o in groppa a un cammello). I cambiamenti effettuati sfociano così in un miglioramento del testo, positivo o negativo che sia. Infatti, ritenuto di frequente in passato l'unico valido criterio traduttivo, fino ad approdare agli eccessi francesi del Sei-Settecento ${ }^{14}$, l'abbellimento viene recentemente osteggiato da Antoine Berman come una delle tante distorsioni cui è da sempre sottoposta la pratica traduttoria nel mondo occidentale ${ }^{15}$. Noi, più modestamente, lo consideriamo solo una delle voci iscritte nel bilancio di ogni traduzione: in questo caso un guadagno, non una perdita.

La situazione inversa, di impoverimento ed entropia, è sempre possibile:

(13) Rinviamo alla definizione di J.P Vinay e J. Dalbernet (Stylistique comparée du français et de l'anglais, Paris, Didier, 1958, p. 61), ripresa, tra gli altri, da Georges Mounin (Teoria e storia della traduzione, Torino, Einaudi, 1965, p. 65) e da Peter
Newmark (op. cit., p. 66).

(14) Cfr. G. Mounin, op. cit., pp. 89-96.

(15) Cfr. il suo saggio La traduction et la lettre ou l'auberge du lointain, Paris, Seuil, 1999. 
«On dit que pour la femme aimée, même la lune est facile à bouger» (p. 93).
«Si dice che chi ama una donna è capace di andarle a prendere anche la luna» (p. 92).

La musicalità di questa sentenza libanese, determinata dalla scansione sillabica di una certa regolarità $(5+10)$, dalle allitterazioni e apofonie ( «pour la femme aimée, même la lune est facile à bouger»), nonché da una specie di rima povera («aimée» / «bouger»), viene sacrificata alla chiarezza del significato che si struttura per allusione alla locuzione italiana «volere la luna». La soluzione scelta, tanto semplificante, si spiega tra l'altro con la necessità di adattare la massima al passo immediatamente successivo, che si origina quasi per associazione di idee, cosicché il nesso non è evidente ed è meglio, per facilitare la lettura, spezzare il paragrafo andando a capo, previo consenso dell'autrice:

«La lune n'a pas eu le temps de finir son tour complet que Salim a recommencé à s'ennuyer» (ibid.).
«La luna non ha fatto in tempo a completare il suo giro che Salim ha ricominciato ad avere nostalgia» (ibid.).

Utile, inoltre, porre mente alla parziale discrepanza fra il lessico finale e le sentenze reperibili all'interno del racconto. Come già detto, molti proverbi, la maggioranza, si ritrovano in tutte e due le parti, mentre alcuni compaiono solo alla fine. Succede anche, seppur sporadicamente, che dei modi di dire riconducibili alla realtà mediorientale che affiorano nel monologo di Dounia vengano invece ignorati nell'elenco ufficiale:

«Je ne suis pas une pastèque qui, en plus de nourrir et d'étancher la soif, sert de repas à l'âne» (p. 125).
«Non sono un cocomero che, oltre a placare la fame e la sete, fa da pasto all'asino» (p. 120).

In ogni modo, ancora una volta, la nostra traduzione si stacca il meno possibile dal testo di partenza.

Riguardo alle sentenze libanesi contenute nel catalogo finale che non figurano, però, nel romanzo, è bene precisare che, come espressamente dichiarato ${ }^{16}$, sono state comunque fonte di ispirazione, per cui si delineano in filigrana dietro questa o quella affermazione fino a determinare, in un caso, un vero e proprio sdoppiamento di ottica: 9).

«Chaque pays a ses coutumes $[\ldots] »(\mathrm{p}$.

«A chaque pays ses usages, à chaque porte sa clé» (p. 175). (p. 17).

«Paese che vai, usanza che trovi $[\ldots] »$ «A ogni paese le sue usanze, a ogni porta la sua chiave» (p. 162).

Per effetto di una specie di «strabismo» linguistico e culturale, al detto tradizionale francese, collocato all'inizio della narrazione, fa da contrappunto quello libanese, presente solo nel lessico, verso la fine. Parallelamente, nel nostro testo all'equivalente italiano si contrappone una versione calcata sull'originale. Che questo tipo di operazione interessi il proverbio in questione, e non un altro, non ci sembra affatto 
casuale: come ogni paese ha le sue usanze e ogni porta la sua chiave, ogni popolo ha la sua identità e, a conferma della bontà del nostro procedere, ogni lingua ha il suo sapore, da gustare e trasmettere.

$\mathrm{Ma}$ - domanda inevitabile - gli adagi popolari dell'edizione italiana, così come noi li abbiamo riformulati, risultano veramente comprensibili a chi legge? A questo riguardo, ci siamo d'istinto affidati alla «conscience-de-proverbe» di cui parla Ber$\operatorname{man}^{17}$, ovverosia abbiamo contato sulla capacità insita in ogni persona di capire intuitivamente un detto straniero ricollegandolo al proprio retaggio proverbiale. Niente note esplicative, quindi, nessun supporto, con una sola eccezione:

«[...] le tarbouche de leur père était encore accroché à la branche du mûrier» (p. 117).
«[...] il fez di loro padre era ancora appeso al ramo del gelso» (p. 112).

Che cosa significhi questa massima non è, a nostro avviso, evidente, per cui ne indichiamo a pie’ pagina il corrispettivo italiano, di solito al singolare, «è un villano rifatto».

Il principio della massima fedeltà ai detti originali, tuttavia, viene da noi rinnegato a sorpresa in una circostanza, peraltro decisamente rilevante:

\section{Le bonbeur a la queue glissante La felicità scivola tra le dita}

Fulcro irradiante dell'opera, nonché suo lasciapassare editoriale, il titolo di una traduzione è sempre il prodotto di una valutazione complessa, ma nel nostro caso ha suscitato parecchie incertezze e ripensamenti. Il testo arabo, che suona più o meno $L i$ scia è la coda della felicità, e l'interpretazione volgare a cui può dar adito il titolo francese («la queue» è anche una designazione volgare del membro virile, qui descritto come «scivoloso») rendevano lecite soluzioni eccentriche, ma di grande effetto quali Sfuggente è la coda della felicità. Potevamo così colpire l'immaginazione del futuro lettore senza - indubbio vantaggio - mancare di coerenza rispetto all'orientamento traduttivo da noi perseguito. Ciononostante, abbiamo preferito in ultima analisi una soluzione più semplice, ma improntata a un'armoniosa eleganza. Questa, infatti, ci sembra meglio rappresentare la composta poliedricità del romanzo, in confronto alla quale risulterebbe invece riduttiva l'animalità esotica di quella «coda sfuggente».

Avviando una prima sintesi, ancora con l'ausilio delle metafore visive di Mounin, prendiamo atto che l'italiano trasparente precedentemente descritto non basta a condurre a buon fine i nostri sforzi. Occorre affiancarvi uno strumento più duttile, che sia capace di acquisire la penetrabilità traslucida dei «vetri colorati» ${ }^{18}$, opponendo all'illusione della traducibilità assoluta la garanzia di un filtro adeguato, in grado di mostrare l'estraneità dell'altra lingua-cultura, evitando di spogliarla delle tinte e sfumature che le sono proprie. Occorre, nella terminologia di Berman, effettuare la trasposizione dello «spirito» nel pieno rispetto della «lettera», ossia tradurre l'uno senza tradire l'altra. 


\section{Conclusione}

Se tralasciamo l'inglese, così poco significativo qui, Le bonbeur a la queue glissante si articola come un caleidoscopio linguistico a tre componenti: il francese standard, il quebecchese, il libanese, di cui il primo è la lente principale su cui si innestano gli altri due. Il francese, infatti, si pone come il frutto di una traduzione preliminare, dal libanese appunto, che appare ora limpida ora invece traslucida, per trasmettere $i$ colori delle altre due realtà linguistiche e culturali. Il francese, insomma è nei termini di Mounin il vetro trasparente che, diventando a tratti opaco, permette di scorgere il quebecchese e soprattutto il dialetto arabo di Dounia, elemento da inquadrare nella sua irriducibile alterità.

Strana combinazione, questa, che coniuga due procedimenti traduttivi da sempre ritenuti distanti se non opposti. Da un lato, l'antica consuetudine di assimilazione del testo straniero, che viene rispettato nei contenuti, ma sottomesso per il resto alle norme e convenzioni della lingua d'arrivo; dall'altro la pratica della massima fedeltà all'originale, di cui si mira a riprodurre non solo il significato, ma anche il particolare sapore linguistico, legato a uno stile, a un'epoca, a una civiltà, a costo di forzare le strutture dell'idioma in cui si traduce ${ }^{19}$. Lì, la pratica etnocentrica di lunga tradizione che mira a riassorbire la diversità, qui la riproduzione letterale che considera l'universo straniero come risorsa, come fonte di arrichimento, secondo una convinzione che è andata affermandosi soprattutto con la modernità, dal romanticismo ai giorni nostri. Fra questi due poli Le bonbeur a la queue glissante cerca e trova una propria mediazione, provando che è possibile modulare anziché scegliere, integrare anziché respingere, in altre parole, conservare la propria identità pur accogliendo l'altro da sé $\mathrm{e}^{20}$.

E noi, che come traduttori arriviamo in seconda battuta, ci troviamo senza dubbio facilitati nell'impresa in quanto, a ben guardare, la strada appare già tracciata, alcuni meccanismi già rodati. Ma proprio per questo, di fronte a un testo che come uno specchio ci rimanda una precisa immagine del nostro lavoro, più vigile dev'essere l'attenzione, maggiore la lucidità. Una traduzione allo specchio, dunque, la nostra, una traduzione di traduzione costretta a guardarsi in faccia e a configurarsi, in risposta a quanto l'originale sembra richiedere, come una superficie che non solo rifletta, ma anche rifranga l'estraneità. Solo così non si perderà il senso profondo di un'opera che, in un mondo globalizzato dove i contatti interculturali non sono più un'opzione, ma un dato di fatto, esorta a mettere la «chiave giusta nella giusta serratura $\gg^{21}$ e aprire la porta, per far entrare lo straniero, lasciandosi affascinare dai suoi modi, dal suo profumo, dal suo accento, senza incorrere nell'accecamento del rifiuto, dell'indifferenza o, peggio, dell'omologazione. In fondo è questo il compito di ogni buon traduttore.

ELETTRA BORDINO ZORZI

(19) Per l'evoluzione storica della teoria della traduzione, consultare i già citati libri di Mounin e Berman e S. BAssnetT, La traduzione. Teorie e pratica, Milano, Bompiani, 1993, cap. 2.
(20) D'altronde è l'obiettivo che si prefigge anche la traduzione «etica, poetica e pensante» di Antoine Berman.

(21) La felicità scivola tra le dita, p. 149. 